\title{
IDENTIFICATION OF THE TeV GAMMA-RAY SOURCE ARGO J2031+4157 WITH THE CYGNUS COCOON
}

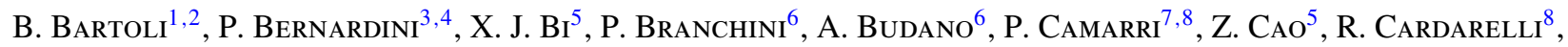 \\ S. Catalanotti ${ }^{1,2}$, S. Z. Chen $^{5,21}$, T. L. Chen $^{9}$, P. Creti ${ }^{4}$, S. W. Cui ${ }^{10}$, B. Z. Dai ${ }^{11}$, A. D'Amone ${ }^{3,4}$, Danzengluobu ${ }^{9}$, \\ I. De Mitri $^{3,4}$, B. D’Ettorre Piazzoli ${ }^{1,2}$, T. Di Girolamo ${ }^{1,2}$, G. Di Sciascio ${ }^{8}$, C. F. Feng ${ }^{12}$, Zhaoyang Feng $^{5}$, \\ ZhenYong FenG ${ }^{13}$, Q. B. Gou ${ }^{5}$, Y. Q. Guo ${ }^{5}$, H. H. He ${ }^{5}$, Haibing Hu ${ }^{9}$, Hongbo Hu ${ }^{5}$, M. Iacovacci ${ }^{1,2}$, R. Iuppa ${ }^{7,8}$, H. Y. Jia ${ }^{13}$, \\ LAbaciren $^{9}$, H. J. Li ${ }^{9}$, G. Liguori ${ }^{14,15}$, C. LiU ${ }^{5}$, J. LiU ${ }^{11}$, M. Y. LiU ${ }^{9}$, H. Lu ${ }^{5}$, L. L. MA ${ }^{5}$, X. H. MA ${ }^{5}$, G. MANCARElla ${ }^{3,4}$, \\ S. M. Mari ${ }^{6,16}$, G. Marsella ${ }^{3,4}$, D. Martello ${ }^{3,4}$, S. Mastroianni ${ }^{2}$, P. Montini ${ }^{6,16}$, C. C. Ning ${ }^{9}$, M. Panareo $^{3,4}$, \\ L. Perrone ${ }^{3,4}$, P. Pistilli ${ }^{6,16}$, F. Ruggieri ${ }^{6}$, P. Salvini ${ }^{15}$, R. Santonico ${ }^{7,8}$, P. R. Shen ${ }^{5}$, X. D. Sheng ${ }^{5}$, F. Shi $^{5}$, A. Surdo $^{4}$, \\ Y. H. TaN ${ }^{5}$, P. Vallania ${ }^{17,18}$, S. Vernetto ${ }^{17,18}$, C. Vigorito ${ }^{18,19}$, H. Wang ${ }^{5}$, C. Y. Wu ${ }^{5}$, H. R. Wu ${ }^{5}$, L. Xue ${ }^{12}$, Q. Y. Yang ${ }^{11}$,

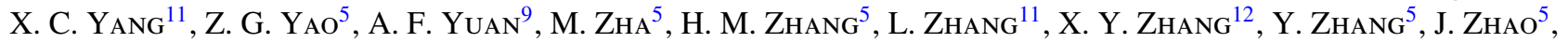

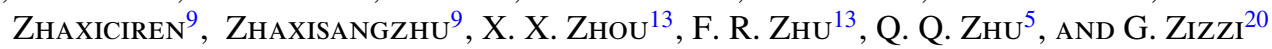 \\ (The ARGO-YBJ Collaboration) \\ ${ }^{1}$ Dipartimento di Fisica dell'Università di Napoli “Federico II," Complesso Universitario di Monte Sant'Angelo, via Cinthia, I-80126 Napoli, Italy \\ ${ }_{2}^{2}$ Istituto Nazionale di Fisica Nucleare, Sezione di Napoli, Complesso Universitario di Monte Sant'Angelo, via Cinthia, I-80126 Napoli, Italy \\ ${ }^{3}$ Dipartimento Matematica e Fisica "Ennio De Giorgi,” Università del Salento, via per Arnesano, I-73100 Lecce, Italy \\ ${ }^{4}$ Istituto Nazionale di Fisica Nucleare, Sezione di Lecce, via per Arnesano, I-73100 Lecce, Italy \\ ${ }^{5}$ Key Laboratory of Particle Astrophysics, Institute of High Energy Physics, Chinese Academy of Sciences, P.O. Box 918, 100049 Beijing, China; chensz@ihep.ac.cn \\ ${ }^{6}$ Istituto Nazionale di Fisica Nucleare, Sezione di Roma Tre, via della Vasca Navale 84, I-00146 Roma, Italy \\ ${ }^{7}$ Dipartimento di Fisica dell'Università di Roma “Tor Vergata," via della Ricerca Scientifica 1, I-00133 Roma, Italy \\ ${ }^{8}$ Istituto Nazionale di Fisica Nucleare, Sezione di Roma Tor Vergata, via della Ricerca Scientifica 1, I-00133 Roma, Italy \\ ${ }^{9}$ Tibet University, 850000 Lhasa, Xizang, P. R. China \\ ${ }^{10}$ Hebei Normal University, Shijiazhuang 050016, Hebei, China \\ ${ }^{11}$ Yunnan University, 2 North Cuihu Road, 650091 Kunming, Yunnan, China \\ ${ }^{12}$ Shandong University, 250100 Jinan, Shandong, P. R. China \\ ${ }^{13}$ Southwest Jiaotong University, 610031 Chengdu, Sichuan, China \\ ${ }^{14}$ Dipartimento di Fisica dell'Università di Pavia, via Bassi 6, I-27100 Pavia, Italy \\ ${ }^{15}$ Istituto Nazionale di Fisica Nucleare, Sezione di Pavia, via Bassi 6, I-27100 Pavia, Italy \\ ${ }^{16}$ Dipartimento di Fisica dell'Università "Roma Tre," via della Vasca Navale 84, I-00146 Roma, Italy \\ ${ }^{17}$ Osservatorio Astrofisico di Torino dell'Istituto Nazionale di Astrofisica, via P. Giuria 1, I-10125 Torino, Italy \\ ${ }^{18}$ Istituto Nazionale di Fisica Nucleare, Sezione di Torino, via P. Giuria 1, I-10125 Torino, Italy \\ ${ }^{19}$ Dipartimento di Fisica dell'Università di Torino, via P. Giuria 1, I-10125 Torino, Italy \\ ${ }^{20}$ Istituto Nazionale di Fisica Nucleare-CNAF, Viale Berti-Pichat 6/2, I-40127 Bologna, Italy \\ Received 2014 May 5; accepted 2014 June 23; published 2014 July 16
}

\begin{abstract}
The extended TeV gamma-ray source ARGO J2031+4157 (or MGRO J2031+41) is positionally consistent with the Cygnus Cocoon discovered by Fermi-LAT at GeV energies in the Cygnus superbubble. Reanalyzing the ARGO-YBJ data collected from 2007 November to 2013 January, the angular extension and energy spectrum of ARGO J2031+4157 are evaluated. After subtracting the contribution of the overlapping TeV sources, the ARGO-YBJ excess map is fitted with a two-dimensional Gaussian function in a square region of $10^{\circ} \times 10^{\circ}$, finding a source extension $\sigma_{\text {ext }}=1.8 \pm 0.5$. The observed differential energy spectrum is $d N / d E=(2.5 \pm 0.4) \times 10^{-11}(E / 1 \mathrm{TeV})^{-2.6 \pm 0.3}$ photons $\mathrm{cm}^{-2} \mathrm{~s}^{-1} \mathrm{TeV}^{-1}$, in the energy range $0.2-10 \mathrm{TeV}$. The angular extension is consistent with that of the Cygnus Cocoon as measured by Fermi-LAT and the spectrum also shows a good connection with the one measured in the 1-100 GeV energy range. These features suggest to identify ARGO J2031+4157 as the counterpart of the Cygnus Cocoon at TeV energies. The Cygnus Cocoon, located in the star-forming region of Cygnus X, is interpreted as a cocoon of freshly accelerated cosmic rays related to the Cygnus superbubble. The spectral similarity with supernova remnants (SNRs) indicates that the particle acceleration inside a superbubble is similar to that in an SNR. The spectral measurements from $1 \mathrm{GeV}$ to $10 \mathrm{TeV}$ allows for the first time to determine the possible spectrum slope of the underlying particle distribution. A hadronic model is adopted to explain the spectral energy distribution.
\end{abstract}

Key words: acceleration of particles - cosmic rays - gamma rays: general

Online-only material: color figures

\section{INTRODUCTION}

The Cygnus region of the Galactic plane stands out in the northern sky for the complex features observed in radio, infrared, X-rays, and gamma-rays. It contains a high density interstellar medium and is rich in potential cosmic ray acceleration

\footnotetext{
${ }^{21}$ Corresponding author: Songzhan Chen.
}

sites, e.g., Wolf-Rayet stars, OB associations, and supernova remnants (SNRs). This region is home of a number of $\mathrm{GeV}$ gamma-ray sources as revealed by Fermi-LAT (Nolan et al. 2012). Several noteworthy $\mathrm{TeV}$ gamma-ray sources were also detected within the Cygnus region in the past decade. A review of these sources can be found in our previous paper (Bartoli et al. 2012a). Gamma-rays are tracers of non-thermal particle acceleration and propagation. The Cygnus region is therefore 
a natural laboratory for studying the origin of cosmic rays, which remains a century-long puzzle.

Cygnus $\mathrm{X}$ in the constellation of Cygnus is the largest starforming region in the solar neighborhood. It contains many distinct $\mathrm{H}$ II regions, a number of Wolf-Rayet stars, and several OB associations, namely OB2, OB1, and OB9. Most objects seen in this region are located at the distance of $\sim 1.4 \mathrm{kpc}$ (Hanson 2003). In the direction of Cyg OB2, the gammaray source TeV J2032+4130 was discovered by the HEGRA collaboration (Aharonian et al. 2002, 2005) and later observed by the Whipple (Konopelko et al. 2007), MAGIC (Albert et al. 2008), and VERITAS (Aliu et al. 2014a) imaging air shower Cherenkov telescopes (IACTs). Its extension is estimated to be about 0.1 and the integral flux above $1 \mathrm{TeV}$ is $3 \%-6 \%$ that of the Crab flux. TeV J2032+4130 is positionally coincident with the GeV pulsar PSR J2032+4127 and could be the corresponding pulsar wind nebula (PWN; Camilo et al. 2009; Aliu et al. 2014a). The sources MGRO J2031+41 and ARGO J2031+4157, detected by Milagro and ARGO-YBJ extensive air shower (EAS) arrays around $20 \mathrm{TeV}$ and $1 \mathrm{TeV}$, respectively, overlap with TeV J2032+4130, but the fluxes are about 10 times higher (Abdo et al. 2007b; Bartoli et al. 2012a, 2013a).

Using the Fermi-LAT data, Ackermann et al. (2011) found a highly extended gamma-ray source above $1 \mathrm{GeV}$, overlapping the $\mathrm{TeV}$ emission. Assuming a symmetrical two-dimensional Gaussian shape, the source angular extension was estimated to be $2.0 \pm 0.2$. The emission was interpreted as a cocoon of freshly accelerated cosmic rays in the Cygnus superbubble, which was taken as evidence for the long-advocated hypothesis that $\mathrm{OB}$ associations host cosmic ray factories. The extended emission region will be denoted as Cygnus Cocoon here.

The extension of MGRO J2031+41, obtained by Milagro with a later analysis, is 1.8 , similar to that of the Cygnus Cocoon. For such a complex region, Abdo et al. (2012a) pointed out that the morphology of MGRO J2031+41 may be produced by the superposition of the central source TeV J2032+4130 and a more extended emission, which causes the large discrepancy between the fluxes measured by IACTs and EAS arrays. However, the correlation between the $\mathrm{TeV}$ extended emission and the Cygnus Cocoon remained unclear.

VERITAS surveyed the Cygnus region. Due to the narrow field of view (FOV; typically 3.5), VERITAS could not detect the gamma-ray emission on the angular scale of the Cygnus Cocoon. In Cherenkov telescopes, the background under a point-like source is estimated by the density of events in rings around the source. For an extended source, this method makes the source to be self-subtracted (Aliu et al. 2013). At one corner of the Cygnus Cocoon, VERITAS detected a new TeV gamma-ray source VER J2019+407 (Aliu et al. 2013) in the direction of SNR G78.2+2.1 with an angular extension of $0.23 \pm 0.05$ and a flux above $320 \mathrm{GeV}$ of $3.7 \% \mathrm{Crab}$ units. A marginal signal associated with VER J2019+407, denoted as ARGO J2021+4038, was reported by the ARGOYBJ collaboration when surveying the northern sky (Bartoli et al. 2013a). This source should also partly contribute to the extended source MGRO J2031+41.

The ARGO-YBJ experiment is an EAS array with a wide FOV, able to monitor the sky in the declination band from $-10^{\circ}$ to $70^{\circ}$ (Bartoli et al. 2013a). Due to the full coverage configuration and the location at high altitude (4300 $\mathrm{m}$ a.s.1.), the detector energy threshold is $\sim 300 \mathrm{GeV}$, much lower than that of Milagro. ARGO-YBJ can extend the measurements of the space-based experiment Fermi-LAT at $0.1-300 \mathrm{GeV}$ to higher energies without any gap. In this paper we reanalyze the ARGOYBJ data recorded in the region of ARGO J2031+4157 to study the relation of this source with the Cygnus Cocoon detected by Fermi-LAT.

\section{THE ARGO-YBJ EXPERIMENT}

The ARGO-YBJ experiment, located at the Yangbajing Cosmic Ray Laboratory (Tibet, China, 90.5 east, 30.1 north), is designed for very high energy (VHE) gamma-ray astronomy (Aielli et al. 2009b, 2009c, 2010; Bartoli et al. 2011a, 2012a, 2012b, 2012c, 2013b) and cosmic ray (Aielli et al. 2009e; Bartoli et al. 2012d, 2012e, 2013c) observations. The detector, extensively described in (Aielli et al. 2006, 2009d), consists of a $74 \times 78 \mathrm{~m}^{2}$ carpet made of a single layer of resistive plate chambers (RPCs) with $\sim 93 \%$ of active area, surrounded by a partially instrumented $(\sim 20 \%)$ "guard ring" area up to $\sim 100 \times$ $110 \mathrm{~m}^{2}$. Each RPC $(2.8 \mathrm{~m} \times 1.25 \mathrm{~m})$ is equipped with 10 logical pixels (called "pads," $55.6 \mathrm{~cm} \times 61.8 \mathrm{~cm}$ ) used for triggering and timing purposes. The arrival times of the particles are measured by 18,360 time-to-digital converters with a resolution of about 1.8 ns (Aielli et al. 2009d), which are calibrated using a software method with precision of 0.4 ns (He et al. 2007; Aielli et al. 2009a).

The ARGO-YBJ experiment in its final configuration started taking data in 2007 November and stopped in 2013 January. The trigger rate is $3.5 \mathrm{kHz}$ with a dead time of $4 \%$ and an average duty-cycle higher than $86 \%$. All the ARGO-YBJ data collected in that period are used in this analysis, for a total observation time of 1670 days. Details of the detector performance and data analysis are widely discussed in Bartoli et al. (2013a), where, using the same data sample, a survey of the northern sky is presented, yielding six sources and five candidates, with an integrated sensitivity of $24 \%$ Crab units. The detector angular resolution for gamma-rays ranges from 1.7 to 0.2 , depending on the number of hit pads $N_{\text {pad }}$ (Bartoli et al. 2011b, 2013a). The sky map in celestial coordinates (right ascension and declination) is divided into a grid of $0.1 \times 0.1$ bins and filled with detected events. The "direct integral method" (Fleysher et al. 2004) is adopted to estimate the number of cosmic ray background events in each bin. A smoothing is applied with an energy-dependent point-spread function (Bartoli et al. 2013a). The Li-Ma method (Li \& Ma 1983) is used to estimate the significance of the excess.

\section{RESULTS}

The significance map around ARGO J2031+4157 as observed by ARGO-YBJ using the events with $N_{\text {pad }} \geqslant 20$ is shown in Figure 1. The highest statistical significance is 6.1 standard deviations (s.ds.), corresponding to the position of ARGO J2031+4157. The positions of other known TeV sources and of the Cygnus Cocoon are also marked in the figure. Our excess almost fully fills the extended region of the Cygnus Cocoon, indicating their similar angular size. At the top right corner of the excess, there is a 4.3 s.d. peak corresponding to ARGO J2021+4038, which is associated with VER J2019+407.

To derive the possible emission from the Cygnus Cocoon, the contribution from the overlapping sources TeV J2032+4130 and VER J2019+407 and from the nearby sources, VER J2016+371 (Aliu et al. 2014b) and MGRO J2019+37 (Abdo et al. 2007b) must be subtracted. For these sources, the spectra and the angular extensions determined in (Aharonian et al. 2005; Aliu et al. 2013, 2014b) are used. For MGRO J2019+37, the flux measured by Milagro is higher than the upper limits derived 


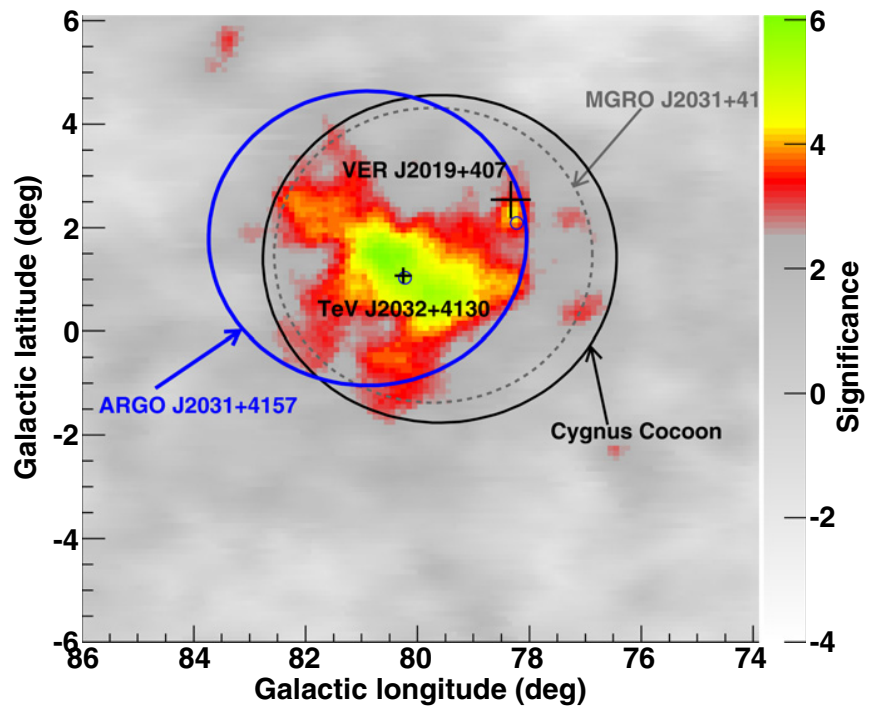

Figure 1. Significance map around ARGO J2031+4157 as observed by the ARGO-YBJ experiment. The large circles indicate the positions of ARGO J2031+4157, MGRO J2031+41, and the Cygnus Cocoon, and the corresponding 68\% containment regions (Ackermann et al. 2011; Abdo et al. 2012a). The position and extension of TeV 2032+4130 and VER J2019+407 are marked with crosses (Aharonian et al. 2005; Aliu et al. 2014b, 2013). The small circles indicate the positions of PSR 2021+4026 and PSR 2032+4127.

(A color version of this figure is available in the online journal.)

by ARGO-YBJ, although still within one s.d. error. Recently, MGRO J2019+37 was resolved into two VERITAS sources, namely VER J2016+371 and VER J2019+368. So the spectra determined by VERITAS (Aliu et al. 2014b), which have better precision and are consistent with both Milagro and ARGO-YBJ measurements, are used here. We track the four sources path inside the ARGO-YBJ FOV and simulate the detector response in the gamma-ray energy range from $10 \mathrm{GeV}$ to $100 \mathrm{TeV}$. The detailed simulation of the ARGO-YBJ detector response to gamma-rays is realized by means of a code based on the GEANT package (Guo et al. 2010). The four sources contributions are removed before estimating the extension and spectrum of the Cygnus Cocoon.

In our previous analysis (Bartoli et al. 2012a), the angular extension of ARGO J2031+4157 was estimated by fitting the angular distribution of the events centered on MGRO J2031+41 within a radius of 2.2 . The excess events outside this region were considered as due to the Galactic diffuse gamma-ray emission. Now, after the Fermi-LAT result indicating the presence of a large extended source, a larger region is used to evaluate the extension of ARGO J2031+4157. To achieve a better angular resolution, only events with $N_{\text {pad }} \geqslant 60$ are used. Assuming a symmetrical two-dimensional Gaussian function for the source shape, we fit the ARGO-YBJ excess in a square region of $10^{\circ} \times 10^{\circ}$ around ARGO $\mathrm{J} 2031+4157$. The result of the fit gives a source position with R.A. $=(307.8 \pm 0.8)^{\circ}$ and decl. $=$ $(42.5 \pm 0.6)^{\circ}$, and an extension $\sigma_{\text {ext }}=(1.8 \pm 0.5)^{\circ}$, consistent with the angular size of the cocoon as measured by Fermi-LAT $(2.0 \pm 0.2)^{\circ}$, within the statistical uncertainties (see Figure 1). The dependence of this result on the source spectral energy distribution is found to be negligible.

To study the spectral behavior of ARGO J2031+4157, the extension $\sigma_{\text {ext }}=2^{\circ}$ and the position of Cygnus Cocoon determined by Fermi-LAT at $1-100 \mathrm{GeV}$ (Ackermann et al. 2011) are used. The fitting method described in Bartoli et al. (2011a) is adopted. In this procedure, the path of the Cygnus

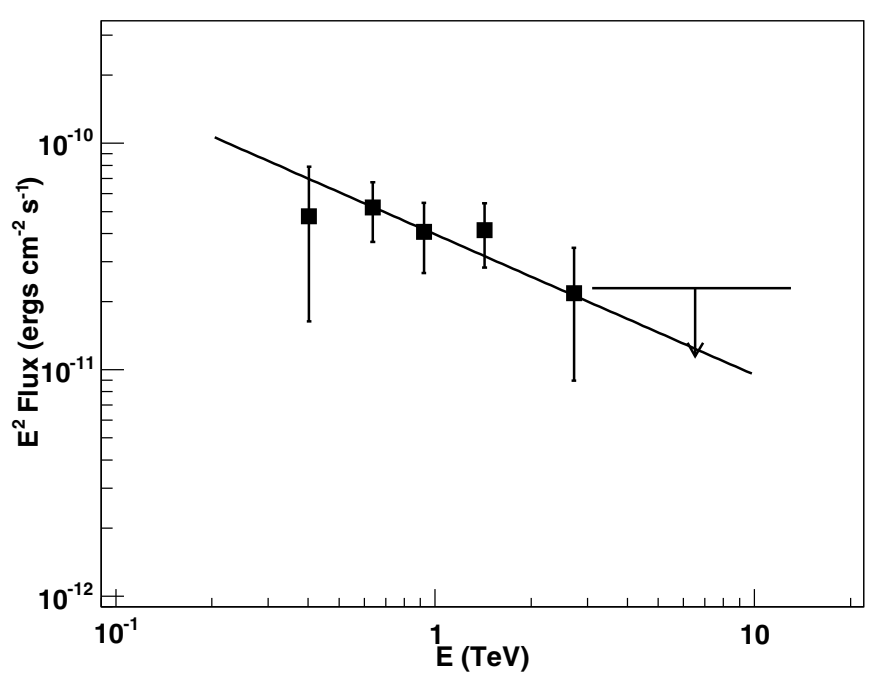

Figure 2. Energy density spectrum of the Cygnus Cocoon as measured by the ARGO-YBJ experiment. The solid line shows the power-law fit to the data points. The arrow indicates the $95 \%$ c.l. upper limit. Only statistical errors are shown.

Cocoon inside the ARGO-YBJ FOV is tracked during the ARGO-YBJ life time. The expected emission is generated by sampling gamma-rays in the energy range $10 \mathrm{GeV}-100 \mathrm{TeV}$ assuming a power-law function. The variable used to determine the event energy is the number of hit pads $N_{\text {pad }}$. The energy spectrum is estimated by comparing the detected signal and the expected signal in six $N_{\text {pad }}$ intervals: 20-39, 40-59, 60-99, 100-199, 200-499, and $\geqslant 500$. Before fitting, the contribution of the four nearby sources is removed. According to our simulations, this contribution is dominated by the two sources, $\mathrm{TeV} \mathrm{J2032+4130} \mathrm{and} \mathrm{VER} \mathrm{J2019+407,} \mathrm{and} \mathrm{is} \mathrm{equal} \mathrm{to} 13.2 \%$, $11.1 \%, 12.1 \%, 10.4 \%$, and $16.2 \%$, respectively, in the first five $N_{\text {pad }}$ intervals (in the sixth interval, there is no excess).

The best fit to the spectrum $\left(\chi^{2} /\right.$ dof $\left.=2.4 / 4\right)$ is

$$
\begin{aligned}
\frac{d N}{d E}= & (2.5 \pm 0.4) \\
& \times 10^{-11}(E / 1 \mathrm{TeV})^{-2.6 \pm 0.3} \text { photons } \mathrm{cm}^{-2} \mathrm{~s}^{-1} \mathrm{TeV}^{-1}
\end{aligned}
$$

The integral flux above $1 \mathrm{TeV}$ is $(1.52 \pm 0.37) \times$ $10^{-11}$ photons $\mathrm{cm}^{-2} \mathrm{~s}^{-1}$, corresponding to $0.8 \pm 0.2 \mathrm{Crab}$ units. The median energies of the six $N_{\text {pad }}$ intervals are $0.40,0.64$, $0.92,1.4,2.7$, and $6.5 \mathrm{TeV}$, respectively. The found spectrum and the corresponding $1 \sigma$ errors are shown in Figure 2. The highest energy point is a 95\% confidence level (c.l.) flux upper limit. The flux is higher than in our previous report (Bartoli et al. 2012a), since a larger source region is considered here. This also gives us a hint that the extension of the source is really larger than our previous estimation. The given errors on the flux are statistical. The systematic errors are estimated to be less than $30 \%$ (Bartoli et al. 2011a).

Note that to subtract the contributions of TeV J2032+4130 and VER J2019+407, the gamma-ray fluxes determined by IACTs are used. Some unclear systematic discrepancy between EAS arrays and IACTs has been found when determining the flux of extended sources. It is worth noting that these two techniques have achieved a good agreement for the point source Crab Nebula (Abdo et al. 2012b; Bartoli et al. 2013a). The fluxes of MGRO J1908+06 and HESS J1841-055 measured by the EAS arrays Milagro and ARGO-YBJ are about two to three 


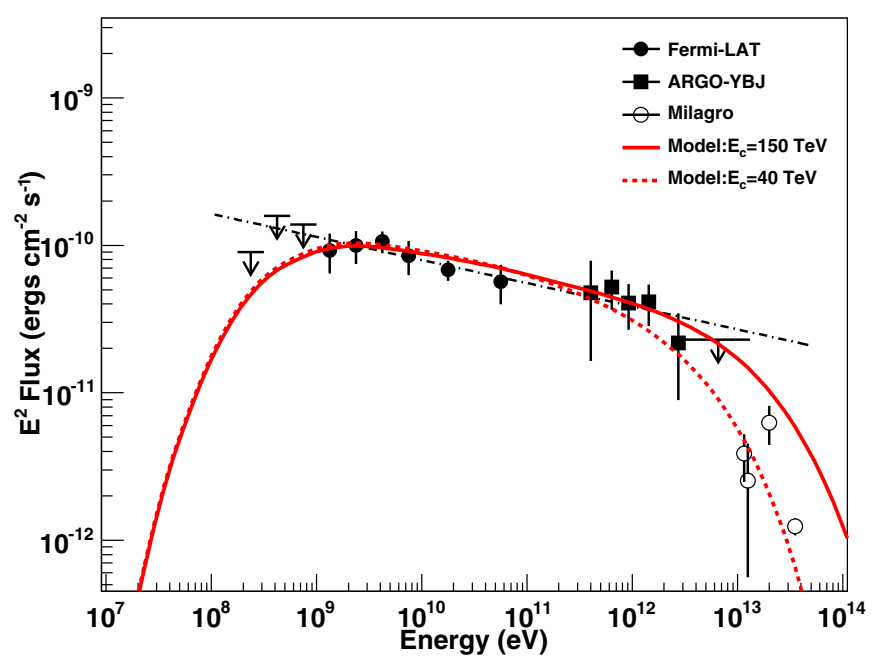

Figure 3. Spectral energy distribution of the Cygnus Cocoon by different detectors. The arrows below $1 \mathrm{GeV}$ indicate the upper limits obtained by FermiLAT (Ackermann et al. 2011). The points at 12, 20, and $35 \mathrm{TeV}$ are reported by Milagro for MGRO J2031+41 (Abdo et al. 2007a, 2007b, 2009). The lower data point at $12 \mathrm{TeV}$ is the Milagro flux after the subtraction of the TeV J2032+4130 contribution (Ackermann et al. 2011). The dot-dashed line shows the best fit to the Fermi-LAT and ARGO-YBJ data using a simple power-law function. The thick solid line is predicted by a hadronic model with a proton cutoff energy at $150 \mathrm{TeV}$. The dotted line is predicted by a model with cutoff energy at $40 \mathrm{TeV}$.

(A color version of this figure is available in the online journal.)

times higher than those determined by IACTs (Bartoli et al. 2012c, 2013b). Therefore we cannot exclude the possibility that the fluxes of TeV J2032+4130 and VER J2019+407 are underestimated by IACTs. In this case, the flux of the Cygnus Cocoon determined here would be overestimated by about 20-30\%. However, the angular sizes of TeV J2032+4130 and VER J2019+407 are smaller than those of MGRO J1908+06 and HESS J1841-055, hence the expected discrepancy should be smaller. In particular, for MGRO J2019+37/VER J2019+368, if we use the Milagro result instead of the VERITAS one, the cocoon flux and extension change by less than $10 \%$.

Figure 3 shows all the spectral measurements by FermiLAT, ARGO-YBJ, and Milagro. The Milagro data refer to the source MGRO J2031+41 (Abdo et al. 2007a, 2007b, 2009), which should contain the contributions from the overlapping and nearby sources. In (Ackermann et al. 2011), the flux of MGRO J2031+41 is corrected by subtracting the extrapolation of TeV J2032+4130 at $12 \mathrm{TeV}$. This "corrected" value is also shown in Figure 3. We should also remind that the Milagro flux at 12 and $20 \mathrm{TeV}$ was determined in a region of $3^{\circ} \times 3^{\circ}$, which is too small compared to the Cygnus Cocoon extension and could contain less than $40 \%$ of the gamma-ray emission. For these reasons, the flux of MGRO J2031+41 is reported in Figure 3 but is not used in the following discussion.

The flux determined by ARGO-YBJ appears consistent with the extrapolation of the Fermi-LAT spectrum. Given the consistency of spectra and angular sizes, the major emission of ARGO J2031+4157 can be identified as the counterpart of the Cygnus Cocoon at $\mathrm{TeV}$ energies. It is worth noting that given the ARGO-YBJ angular resolution, a detailed comparison with the morphology found by Fermi-LAT is meaningless.

The combined spectrum of Fermi-LAT and ARGO-YBJ is fitted $\left(\chi^{2} /\right.$ dof $\left.=2.7 / 9\right)$ by the power-law function $d N / d E=$ $(3.5 \pm 0.3) \times 10^{-9}(E / 0.1 \mathrm{TeV})^{-2.16 \pm 0.04}$ photons $\mathrm{cm}^{-2} \mathrm{~s}^{-1} \mathrm{TeV}^{-1}$, as shown by the dot-dashed line in Figure 3. The upper limits of Fermi-LAT and ARGO-YBJ indicate weak evidence for the presence of a slope change or cutoff below $\sim 1 \mathrm{GeV}$ and above $\sim 10 \mathrm{TeV}$, respectively.

\section{DISCUSSION}

The angular size of about $2^{\circ}$ places the Cygnus Cocoon among the most extended VHE gamma-ray sources. At a distance of $1.4 \mathrm{kpc}$, the observed angular extension corresponds to more than $50 \mathrm{pc}$, making the Cygnus Cocoon the largest identified Galactic $\mathrm{TeV}$ source. Such a large region can be related to different scenarios. PWNs and SNRs contribute to most of the extended Galactic TeV sources identified up to now. Toward the Cygnus Cocoon, two pulsars (PSR J2021+4026 and PSR J2032+4127) and one SNR (SNR G78.2+2.1) have been detected. As remarked on by Ackermann et al. (2011), the PWNs powered by these two pulsars are unlikely to explain the cocoon emission and SNR G78.2+2.1 could be too young to be the unique accelerator in the cocoon able to diffuse over the whole region. However, PSR J2032+4127 and the Cygnus Cocoon are well-coincident apparently, and we cannot rule out the possibility that the cocoon emission is from the yet undiscovered remnant of a supernova that originated the pulsar. The favored scenario of Ackermann et al. (2011) is the injection of cosmic rays via acceleration from the collective action of multiple shocks from supernovae and winds of massive stars, which form the Cygnus superbubble. Such superbubbles have been long advocated as cosmic ray factories (Bykov \& Toptygin 2001; Parizot et al. 2004; Ferrand \& Marcowith 2010). Possibly, the Cygnus Cocoon is the first evidence supporting such a hypothesis.

For such a large extended region, no significant morphology and spectrum variation have been found by Ackermann et al. (2011) in the range from 1 to $100 \mathrm{GeV}$. The energy spectrum from $1 \mathrm{GeV}$ to $10 \mathrm{TeV}$ shows a simple power-law shape, which is very similar to those of SNRs, such as Cassiopeia A, Tycho, W51C, IC 443, and so on (Yuan et al. 2012). This indicates that the Cygnus Cocoon may be an unknown SNR, or that the particle acceleration inside a superbubble is similar to that in an SNR. No matter which accelerator is responsible for the Cygnus Cocoon emission, the whole spectral shape of the gamma-ray emission from $1 \mathrm{GeV}$ to $10 \mathrm{TeV}$ allows us to determine a possible spectral slope of the underlying particle distribution for the first time. Different scenarios have been proposed to explain the emission mechanism of gamma-rays, which can be produced via inverse Compton (IC) scattering of background photon fields by high-energy electrons or, in hadronic models, by $\pi_{0}$ decay from inelastic proton-proton or proton-photon interactions. The electron bremsstrahlung can be ignored if the electron-to-proton ratio is about $1 \%$ as measured around Earth. The close relation between the emission morphology and the interstellar structure revealed by Ackermann et al. (2011) favors a cosmic ray origin. The Fermi-LAT measurement below $3 \mathrm{GeV}$ is also a hint of the $\pi_{0}$ decay feature Ackermann et al. (2013). Moreover, the gamma-ray spectrum predicted by IC process is always curved, and it is difficult for the pure leptonic model to produce such a simple power-law shape from $1 \mathrm{GeV}$ to $10 \mathrm{TeV}$.

In this work, we adopt a purely hadronic emission model (Drury et al. 1994) to produce the gamma-ray emission from the cocoon. In our model, the observed gamma-rays are attributed to the decay of $\pi_{0}$ mesons produced in inelastic collisions between accelerated protons and target gas. The predicted spectrum is shown as the thick solid line in Figure 3. It is assumed that the primary proton spectrum follows a power law with index $\alpha$ and with an exponential cutoff energy $E_{c}$, i.e., $E^{\alpha} e^{-E / E_{c}}$. The value 
of $\alpha$ is the same as the spectral index of gamma-rays and $E_{c}$ is set to $150 \mathrm{TeV}$. This $E_{c}$ value is the maximum allowed by the ARGO-YBJ upper limit. It is worth recalling that as explained in the last section, the data points obtained by Milagro are not taken into account for this model. Taking them into account, the $E_{c}$ would be around $40 \mathrm{TeV}$, giving the dotted line shown in Figure 3. The total energy of the high-energy protons is $1.5 \times 10^{50} \times(10 / n)$ erg at a distance of $1.4 \mathrm{kpc}$, where $n$ is the gas density per $\mathrm{cm}^{3}$ averaged over the emission volume. The Cygnus region hosts a giant molecular cloud complex with total mass of $8 \times 10^{6} M_{\odot}$ (Ackermann et al. 2012). The interstellar gas density should be higher than $10 \mathrm{~cm}^{-3}$. Hence, the derived proton power should be less than $1.5 \times 10^{50} \mathrm{erg}$, which can be reasonably provided by one supernova, which usually releases $\sim 10^{51} \mathrm{erg}$, about $10 \%$ of which can be transferred to the accelerated particles. This result plausibly supports one SNR or several SNRs, i.e., a supperbubble, as the accelerator responsible for the Cygnus Cocoon emission. The main feature of the gammaray emission from the hadronic model is that it predicts a clear cutoff at energy below $1 \mathrm{GeV}$ Ackermann et al. (2013), which is significantly different from that from the leptonic model and can be checked by future long-term observation from Fermi-LAT.

In a cosmic ray source, both electrons and nuclei can be accelerated at the same time. The gamma-ray spectrum of Cygnus Cocoon can also be reproduced by a hybrid model. Recently, Yuan et al. (2012) proposed a unified model for the gamma-ray emission of SNRs. In this model, they assumed a constant ratio (1\%) and identical spectral shape for cosmicray protons and electrons, which were derived from the locally observed spectra by taking into account the transport effects from sources to the observers. The diversity of gamma-ray spectra can be caused only by the differences of the medium gas density. According to this model, when the differential spectral index of gamma-rays is 2.16 , the gamma-ray emission below $100 \mathrm{GeV}$ is dominated by pion decay and the emission above $100 \mathrm{GeV}$ is dominated by the electron inverse Compton scattering on low-energy background photons.

\section{CONCLUSIONS}

Using the whole ARGO-YBJ data collected from 2007 November to 2013 January, we reanalyzed the TeV gammaray emission from ARGO J2031+4157. The source extension is found consistent with that of the Cygnus Cocoon determined by Fermi-LAT at 1-100 GeV. The measured spectrum is consistent with a simple power-law extrapolation of the spectrum measured by Fermi-LAT, suggesting the same origin for both $\mathrm{GeV}$ and TeV gamma-ray emissions. These features support the identification of ARGO J2031+4157 as the TeV counterpart of the Cygnus Cocoon.

Even if a yet undiscovered nebula cannot be ruled out, such a large extended emission is likely due to the collective action of multiple shocks in a superbubble. The broad spectrum and the similarity with other SNRs indicate that the particle acceleration inside a superbubble is similar to that in an SNR. The GeV-TeV spectrum allows for the first time to determine the possible spectral slope of the underlying particle distribution. To further investigate into the Cygnus Cocoon and its relation with TeV J2032+4130, more sensitive observations by detectors with large collection area, high angular resolution, and wide FOV are needed. The new EAS experiments, such as HAWC, Tibet+MD, and LHAASO (more details about these detectors can be found in Chen 2013) are expected to play an important role for such an issue.

This work is supported in China by NSFC (No. 10120130794, No. 11205165, No. 11375210), the Chinese Ministry of Science and Technology, the Chinese Academy of Sciences, the Key Laboratory of Particle Astrophysics, CAS, and in Italy by the Istituto Nazionale di Fisica Nucleare (INFN). We also acknowledge the essential support of W. Y. Chen, G. Yang, X. F. Yuan, C. Y. Zhao, R. Assiro, B. Biondo, S. Bricola, F. Budano, A. Corvaglia, B. D’Aquino, R. Esposito, A. Innocente, A. Mangano, E. Pastori, C. Pinto, E. Reali, F. Taurino, and A. Zerbini in the installation, debugging, and maintenance of the detector.

\section{REFERENCES}

Abdo, A. A., Abeysekara, U., Allen, B. T., et al. 2012a, ApJ, 753, 159 Abdo, A. A., Allen, B., Berley, D., et al. 2007a, ApJL, 658, L33 Abdo, A. A., Allen, B., Berley, D., et al. 2007b, ApJL, 664, L91 Abdo, A. A., Allen, B., Berley, D., et al. 2009, ApJL, 700, L127 Abdo, A. A., Allen, B. T., Atkins, R., et al. 2012b, ApJ, 750, 63 Ackermann, M., Ajello, M., Allafort, A., et al. 2011, Sci, 334, 1103 Ackermann, M., Ajello, M., Allafort, A., et al. 2012, A\&A, 538, 71 Ackermann, M., Ajello, M., Allafort, A., et al. 2013, Sci, 339, 807 Aharonian, F., Akhperjanian, A. G., Beilicke, M., et al. 2002, A\&A, 393, L37 Aharonian, F., Akhperjanian, A. G., Beilicke, M., et al. 2005, A\&A, 431, 197 Aielli, G., Assiro, R., Bacci, C., et al. 2006, Nucl. Instrum. Meth. A, 562, 92 Aielli, G., Assiro, R., Bacci, C., et al. 2009a, APh, 30, 287

Aielli, G., Bacci, C., Bartoli, B., et al. 2009b, APh, 32, 47

Aielli, G., Bacci, C., Bartoli, B., et al. 2009c, ApJ, 699, 1281

Aielli, G., Bacci, C., Bartoli, B., et al. 2009d, Nucl. Instrum. Meth. A, 608, 246 Aielli, G., Bacci, C., Bartoli, B., et al. 2009e, PhRvD, 80, 092004

Aielli, G., Bacci, C., Bartoli, B., et al. 2010, ApJL, 714, L208

Albert, J., Aliu, E., Anderhub, H., et al. 2008, ApJL, 675, L25 Aliu, E., Archambault, S., Arlen, T., et al. 2013, ApJ, 770, 93 Aliu, E., Aune, T., Behera, B., et al. 2014a, ApJ, 783, 16 Aliu, E., Aune, T., Behera, B., et al. 2014b, ApJ, 788, 78 Bartoli, B., Bernardini, P., Bi, X. J., et al. 2011a, ApJ, 734, 110 Bartoli, B., Bernardini, P., Bi, X. J., et al. 2011b, PhRvD, 84, 022003 Bartoli, B., Bernardini, P., Bi, X. J., et al. 2012a, ApJL, 745, L22 Bartoli, B., Bernardini, P., Bi, X. J., et al. 2012b, ApJ, 758, 2 Bartoli, B., Bernardini, P., Bi, X. J., et al. 2012c, ApJ, 760, 110 Bartoli, B., Bernardini, P., Bi, X. J., et al. 2012d, PhRvD, 85, 022002 Bartoli, B., Bernardini, P., Bi, X. J., et al. 2012e, PhRvD, 85, 092005 Bartoli, B., Bernardini, P., Bi, X. J., et al. 2013a, ApJ, 779, 27 Bartoli, B., Bernardini, P., Bi, X. J., et al. 2013b, ApJ, 767, 99 Bartoli, B., Bernardini, P., Bi, X. J., et al. 2013c, PhRvD, 88, 082001 Bykov, A. M., \& Toptygin, I. N. 2001, AstL, 27, 625 Camilo, F., Ray, P. S., Ransom, S. M., et al. 2009, ApJ, 705, 1 Chen, S. Z. 2013, Sci. China Phys. Mechan. Astron., 56, 1454 Drury, L. O'C, Aharonian, F., \& Völk, H. J. 1994, A\&A, 287, 959

Ferrand, G., \& Marcowith, A. 2010, A\&A, 510, 101

Fleysher, R., Fleysher, L., Nemethy, P., \& Mincer, A. I. 2004, ApJ, 603, 355 Guo, Y. Q., Zhang, X. Y., Zhang, J. L., et al. 2010, ChPhC (HEP \& NP), 34, 555 Hanson, M. M. 2003, ApJ, 597, 957

He, H. H., Bernardini, P., Calabrese Melcarne, A. K., \& Chen, S. Z. 2007, APh, 27,528

Konopelko, A., Atkins, R. W., Blaylock, G., et al. 2007, ApJ, 658, 1062

Li, T. P., \& Ma, Y. Q. 1983, ApJ, 272, 317

Nolan, P. L., Abdo, A. A., Ackermann, M., et al. 2012, ApJS, 199, 31

Parizot, E., Marcowith, A., van der Swaluw, E., Bykov, A. M., \& Tatischeff, V. 2004, A\&A, 424, 747

Yuan, Q., Liu, S. M., \& Bi, X. J. 2012, ApJ, 761, 133 